\title{
Perioperative atrial tachycardia is associated with increased mortality in infants undergoing cardiac surgery
}

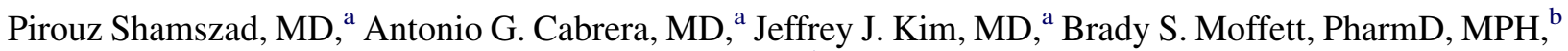 \\ Daniel E. Graves, PhD, MEd, ${ }^{\mathrm{c}}$ Jeffrey S. Heinle, MD, ${ }^{\mathrm{d}}$ and Joseph W. Rossano, MD
}

\begin{abstract}
Objective: Few data are available on the frequency or importance of perioperative atrial tachycardia in infants. We hypothesized that atrial tachycardia in infants undergoing cardiac surgery is not rare and is associated with increased morbidity and mortality.
\end{abstract}

\begin{abstract}
Methods: From 2007 through 2010, 777 infants (median age, 1.8 months; interquartile range, 0.33-5.73) underwent cardiac surgery. Their medical records were reviewed for atrial tachycardia during the perioperative period.

Results: Of the 777 patients, 64 (8.2\%) developed atrial tachycardia. The independent risk factors for developing atrial tachycardia included surgical age 6 months or younger (odds ratio, 4.4; 95\% confidence interval, 1.1-19.15), use of 3 or more inotropes (odds ratio, 2.9; 95\% confidence interval, 1.4-6.2), and heterotaxy syndrome (odds ratio, $2.9 ; 95 \%$ confidence interval, 1.1-7.4). All-cause mortality in the atrial tachycardia group was increased $(21.9 \%$ vs $7.2 \%, P<.001)$ during a median follow-up period of 14.6 months (interquartile range, 6.8-24.6), and atrial tachycardia was independently associated with decreased survival (hazard ratio, 1.9; 95\% confidence interval, 1.1-3.8). Infants with perioperative atrial tachycardia had a longer hospital length of stay (32 vs 17 days, $P<.001$ ) and duration of inotrope use (10.5 vs 3.0 days, $P<.001)$. A total of 57 patients received antiarrhythmic therapy, with propranolol the most common $(\mathrm{n}=31)$. Among the survivors, 48 patients received outpatient antiarrhythmic therapy, which was successfully discontinued in 23 patients at a median duration of 14 months (interquartile range, 5.7-18.6) without recurrence.
\end{abstract}

Conclusions: Atrial tachycardia is common in infants undergoing cardiac surgery and is independently associated with decreased survival. Among survivors, antiarrhythmic agents successfully controlled atrial tachycardia in most patients with a low recurrence risk after discontinuation. (J Thorac Cardiovasc Surg 2012;144:396-401)

Earn CME credits at

http://cme.ctsnetjournals.org factors have all been postulated as risk factors for increased rates of postoperative arrhythmia. ${ }^{1,4-6}$

Focal atrial tachycardia (AT) is a form of supraventricular arrhythmia that occurs in infants with and without congenital heart disease..$^{7-10}$ Although previous reports have described longer hospital stays, intubation times, and requirement for inotropic support in infants with perioperative cardiac arrhythmias, ${ }^{2,11}$ only limited data have described the risk factors and outcome of perioperative AT in infants undergoing cardiac surgery. Furthermore, the mortality associated with AT has not been reported in this age group. Therefore, the present study aimed to test the hypothesis that AT in infants undergoing cardiac surgery is not a rare occurrence and is associated with increased morbidity and mortality. The risk factors associated with AT were also assessed.

\section{METHODS}

A retrospective review of the pediatric cardiovascular surgery database identified all infants who underwent cardiac surgery at Texas Children's Hospital from July 2007 through July 2010. The medical records of these infants were then reviewed in the pediatric cardiology database to identify those diagnosed with AT within the perioperative period. The Baylor College of Medicine institutional review board approved the study. 


\author{
Abbreviations and Acronyms \\ $\mathrm{AT}=$ atrial tachycardia \\ $\mathrm{CI}=$ confidence interval \\ $\mathrm{ECMO}=$ extracorporeal membrane oxygenation \\ $\mathrm{IQR}=$ interquartile range \\ $\mathrm{OR} \quad=$ odds ratio
}

\section{Patient Selection}

All infants who underwent cardiac surgery during the study period were included. This included all infants with structural heart disease and those who required placement of a ventricular assist device or cardiac transplantation. We excluded infants who underwent pacemaker implantation for congenital atrioventricular block, pericardial window creation for recurrent pericardial effusion, or extracorporeal membrane oxygenation cannulation for cardiopulmonary failure without concomitant cardiac surgery. All infants were admitted to the cardiac intensive care unit postoperatively, received continuous cardiac telemetry during their intensive care unit stay, and had at least 1 electrocardiogram recorded postoperatively. The medical records were reviewed for cases of AT. All cases were confirmed by a pediatric electrophysiologist. Macroreentrant AT such as atrial flutter and fibrillation was not included.

\section{Definitions}

Infancy was defined as patient age younger than 12 months. The perioperative period spanned 10 days preoperatively to 30 days postoperatively. The diagnosis and procedural variables were stratified using the Risk Adjustment for Congenital Heart Surgery scoring system. ${ }^{12}$

AT was defined as 3 or more consecutive beats of tachycardia originating from an alternative atrial focus different than sinus. To be included in the present study, the degree of AT was considered to be of enough clinical significance by the attending cardiologist of record to require therapy alteration, including initiation of antiarrhythmic treatment or close electrocardiographic observation. For the purposes of the present study, because single 3-beat runs of tachycardia were typically not treated, they were not included in the present study. Features aiding in the identification of AT included an acute change in P wave morphology during tachycardia at a rate at least $20 \%$ faster than sinus. In cases of heterotaxy syndrome, care was taken to accurately identify AT in the presence of more than 1 primary atrial rhythm. A review of inpatient electrocardiographic telemetry or 24-hour Holter monitor data was performed independently by a pediatric cardiac electrophysiologist (J.J.K.) to confirm the diagnosis. A representative 12-lead electrocardiogram and atrial electrocardiogram are displayed

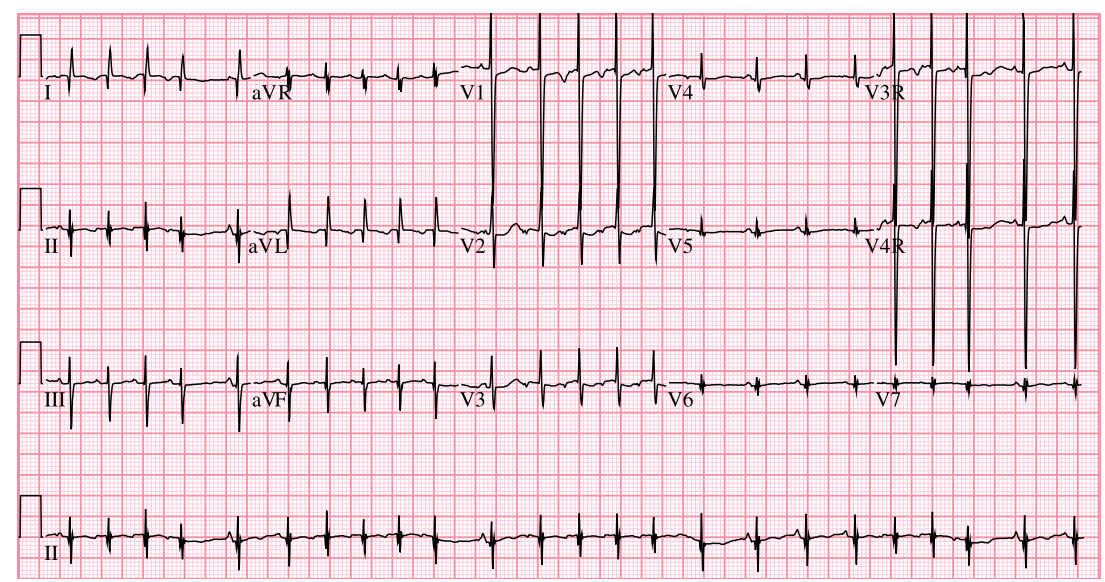

A

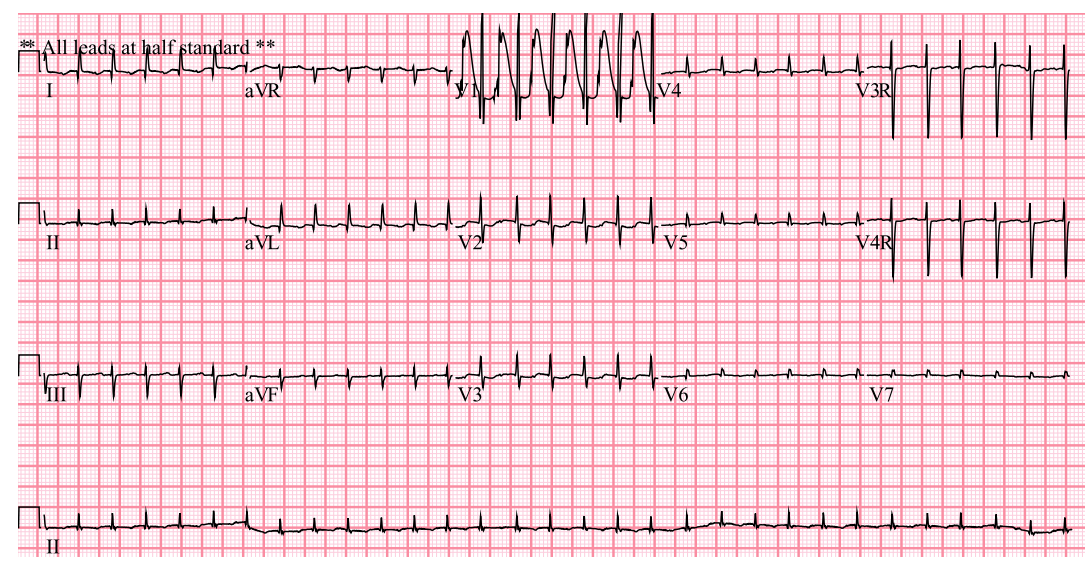

B

FIGURE 1. A, Postoperative 12-lead electrocardiogram of an infant with atrial tachycardia. B, Postoperative atrial electrocardiogram (attached to lead $V_{1}$ ) of an infant with atrial tachycardia. 
in Figure 1, $A$ and $B$, respectively. Patients diagnosed with AT during the perioperative period were assigned to the study group, and all other patients were assigned to the control group. The choice of management, including type of antiarrhythmic agent, was at the sole discretion of the attending cardiologist of record at the onset of AT.

\section{Data Collection and Statistical Analysis}

The preoperative and postoperative data were obtained by a review of medical records at Texas Children's Hospital. The intraoperative data were obtained from the pediatric cardiovascular surgery database. Univariable analysis of categorical variables was performed using Pearson's chi-square or Fisher's exact test, as appropriate. Continuous variables were analyzed using the Mann-Whitney $U$ test because the data were not normally distributed. Survival analysis was performed using KaplanMeier estimation. Factors significantly associated with the development of AT and mortality were subsequently analyzed in multivariable analyses. Multivariable binary logistic regression analysis was used to determine the independent risk factors for developing AT, and Cox regression analysis was used to determine the independent risk factors for mortality. A 2-tailed test of significance was used for all statistical analyses.

\section{RESULTS}

\section{Patient Population}

A total of 777 infants at a median age of 1.8 months (interquartile range [IQR], 0.33-5.73) who underwent cardiac surgery at Texas Children's Hospital during the study period were included. Of those, 64 infants $(8.2 \%)$ were diagnosed with AT. Significant residual cardiac lesions requiring reoperation or catheter-based intervention within the postoperative study period was relatively uncommon and was present in 19 patients $(2.4 \%, 5$ in the AT group). The common diagnoses and patient characteristics of both groups are listed in Table 1. AT was more common in Hispanics $(P=.02)$. The median age and weight at surgery were significantly lower for patients with AT than for the control group $(P<.001$ and $P<.001$, respectively).

\section{Presence of AT}

Of the 777 patients, $64(8.2 \%)$ were diagnosed with AT and were included in the study group. Of these 64 patients, $8(12.5 \%)$ developed AT at a median of 4.0 days (IQR, 0-6.0) preoperatively, and $56(87.5 \%)$ developed AT at a median of 8.5 days (IQR, 3.0-14.0) postoperatively. The mean maximal rate of tachycardia was 241.2 beats $/ \mathrm{min}$ (range, 160-369) at a median of 7.0 days (IQR, $0.25-12.0)$ postoperatively. The factors associated with the development of AT on univariable and multivariable analysis are listed in Table 2. On multivariable analysis, heterotaxy syndrome, age at surgery younger than 6 months, the use of 3 or more inotropes, total anomalous pulmonary venous return, and transposition of the great arteries were independent risk factors for the development of AT.

On univariable analysis, the specific inotropes used during the perioperative period were associated with an increased risk of postoperative AT. The preoperative use of dopamine (odds ratio [OR], 2.97; 95\% confidence
TABLE 1. Patient demographics

\begin{tabular}{|c|c|c|c|}
\hline Demographic & $\begin{array}{c}\text { No AT } \\
(n=713)\end{array}$ & $\begin{array}{c}\text { AT } \\
(n=64)\end{array}$ & $P$ value \\
\hline Age (mo) & & & $<.001$ \\
\hline Median & 2.37 & 0.27 & \\
\hline IQR & $0.39-6.00$ & $0.20-1.23$ & \\
\hline Male gender (n) & $391(54.8)$ & $42(65.6)$ & .06 \\
\hline \multicolumn{4}{|l|}{ Ethnicity (n) } \\
\hline Asian & $25(3.5)$ & $1(1.6)$ & NS \\
\hline Black & $99(13.9)$ & $9(14.1)$ & NS \\
\hline Hispanic & $262(36.7)$ & 33 (51.6) & .02 \\
\hline White & $327(45.9)$ & $21(32.8)$ & .03 \\
\hline Prematurity (n) & $153(21.5)$ & $15(23.4)$ & NS \\
\hline Birth weight $(\mathrm{kg})$ & & & NS \\
\hline Median & 3.0 & 3.0 & \\
\hline IQR & $2.59-3.35$ & $2.71-3.39$ & \\
\hline Weight at surgery $(\mathrm{kg})$ & & & $<.001$ \\
\hline Median & 4.0 & 3.2 & \\
\hline IQR & $3.11-6.10$ & $2.79-3.72$ & \\
\hline Single ventricle anatomy (n) & $150(21.0)$ & $25(39.1)$ & $<.001$ \\
\hline RACHS-1 score $\geq 4$ (n) & $198(27.8)$ & $37(57.8)$ & $<.001$ \\
\hline \multicolumn{4}{|l|}{ Diagnosis (n) } \\
\hline Ventricular septal defect & $105(14.7)$ & $2(3.1)$ & $<.001$ \\
\hline Tetralogy of Fallot & 83 (11.6) & $2(3.1)$ & .02 \\
\hline Atrioventricular septal defect & $73(10.2)$ & $3(4.7)$ & NS \\
\hline Hypoplastic left heart syndrome & $55(7.7)$ & $10(15.6)$ & .03 \\
\hline Coarctation of aorta & $49(6.9)$ & $2(3.1)$ & NS \\
\hline Transposition of great arteries & $38(5.3)$ & $9(14.1)$ & .01 \\
\hline Heterotaxy syndrome & $33(4.6)$ & $11(17.2)$ & $<.001$ \\
\hline $\begin{array}{l}\text { Total anomalous pulmonary } \\
\text { venous return }\end{array}$ & $29(4.1)$ & $7(10.9)$ & .02 \\
\hline Cardiopulmonary bypass time (min) & $\mathrm{n}=530$ & $\mathrm{n}=53$ & NS \\
\hline Median & 168.5 & 182 & \\
\hline IQR & $121-216$ & $135.5-231$ & \\
\hline Aortic crossclamp time (min) & $\mathrm{n}=508$ & $\mathrm{n}=51$ & NS \\
\hline Median & 96 & 94 & \\
\hline IQR & $69.3-129$ & $67-139$ & \\
\hline $\begin{array}{l}\text { Antegrade cerebral perfusion time } \\
\text { (min) }\end{array}$ & $\mathrm{n}=113$ & $\mathrm{n}=27$ & NS \\
\hline Median & 56 & 66 & \\
\hline IQR & $25-84$ & $37.8-79.8$ & \\
\hline $\begin{array}{l}\text { Deep hypothermic circulatory arrest } \\
\text { time (min) }\end{array}$ & $\mathrm{n}=115$ & $\mathrm{n}=16$ & $<.001$ \\
\hline Mean & 13 & 18 & \\
\hline IQR & $8-23$ & $8-39$ & \\
\hline
\end{tabular}

Data in parentheses are percentages. $A T$, Atrial tachycardia; $I Q R$, interquartile range; RACHS-1, Risk Adjustment for Congenital Heart Surgery.

interval [CI], 1.17-7.56) or calcium chloride (OR, 3.38; 95\% CI, 1.4-8.15) was significantly associated with an increased risk of AT. Postoperatively, epinephrine (OR, 5.59; 95\% CI, 2.98-10.12), vasopressin (OR, 3.37; 95\% CI, 2.01-5.67), and calcium chloride (OR, 4.36; $95 \%$ CI, 2.51-7.57) use were significant. Milrinone did not significantly contribute any additive risk to the development of AT. Although no individual inotrope was significantly associated with the development of AT on multivariable 
TABLE 2. Risk factors for developing AT

\begin{tabular}{|c|c|c|c|c|}
\hline \multirow[b]{2}{*}{ Factor } & \multicolumn{2}{|c|}{$\begin{array}{c}\text { Univariable } \\
\text { analysis }\end{array}$} & \multicolumn{2}{|c|}{$\begin{array}{l}\text { Multivariable } \\
\text { analysis }\end{array}$} \\
\hline & OR & $95 \%$ CI & OR & $95 \%$ CI \\
\hline Age $\leq 6$ mo at surgery & 10.47 & $2.54-43.23$ & 4.41 & $1.05-19$. \\
\hline $\begin{array}{l}\text { Total anomalous pulmonary } \\
\text { venous return }\end{array}$ & 90 & 0 & 3.07 & 3.50 \\
\hline Use of $\geq 3$ inotropes & 5.92 & $3.33-10.54$ & 2.91 & $1.37-6.19$ \\
\hline Heterotaxy syndrome & 4.28 & $2.05-8.94$ & 2.89 & $1.13-7.39$ \\
\hline Transposition of grea & 2.91 & $1.34-6.32$ & 2.66 & $1.10-6.42$ \\
\hline Single ventricle physiology & 2.41 & $1.41-4.10$ & 1.64 & $0.76-3.57$ \\
\hline Hispanic & 1.83 & $1.10-3.06$ & 1.56 & $0.89-2.75$ \\
\hline RACHS- 1 score $\geq 4$ & 3.76 & $2.21-6.41$ & 1.55 & $0.78-2.95$ \\
\hline Use of preoperative in & 2.17 & $1.13-4.17$ & 1.03 & $0.48-2.21$ \\
\hline Hypoplastic left heart syndrome & 2.22 & $1.07-4.59$ & 0.90 & $0.32-2.52$ \\
\hline Use of postoperative inotropes & 2.55 & $1.12-5.69$ & 0.81 & $0.31-2.09$ \\
\hline
\end{tabular}

$O R$, Odds ratio; $C I$, confidence interval; $R A C H S-1$, Risk Adjustment for Congenital Heart Surgery.

analysis, the use of 3 or more inotropes was significant (OR, 2.91; 95\% CI, 1.37-6.19; Table 2).

\section{Patient Outcomes}

Patients with AT had longer hospital stays and inotrope durations compared with the non-AT group (Table 3). A total of 15 patients $(1.9 \%), 10$ control patients and 5 study patients $(P=.01)$, required extracorporeal membrane oxygenation (ECMO) support during the postoperative period owing to hemodynamic instability of differing etiologies. Incessant AT was the indication for ECMO in only 1 neonate, who initially required 72 hours of support, but went on to an uneventful discharge home. Although the remaining 4 study patients developed AT sometime during the perioperative period after ECMO decannulation, the indication for ECMO postoperatively was an acute, nonarrhythmic cause of hemodynamic compromise.

The overall mortality of the study group was $21.9 \%$ versus $7.2 \%$ in the control group $(P<.001)$ during the follow-up period. The median follow-up was 12.3 months (IQR, 4.5-24.5) in the AT group and 14.7 months

TABLE 3. Patient outcomes

\begin{tabular}{|c|c|c|c|}
\hline Demographic & $\begin{array}{c}\text { No AT } \\
(n=713)\end{array}$ & $\begin{array}{c}\text { AT } \\
(n=64)\end{array}$ & $P$ value \\
\hline Overall all-cause mortality (n) & $51(7.2)$ & $14(21.9)$ & $<.001$ \\
\hline 30-d Postoperative mortality (n) & $13(1.8)$ & $3(4.7)$ & NS \\
\hline 30-d Readmission (n) & $82(11.5)$ & $5(7.8)$ & NS \\
\hline Hospital length of stay (d) & & & $<.001$ \\
\hline Median & 17 & 32 & \\
\hline IQR & $9-31$ & $19.8-69.5$ & \\
\hline Inotrope duration (d) & & & $<.001$ \\
\hline Median & 3 & 10.5 & \\
\hline IQR & $1-8$ & $6-26.5$ & \\
\hline Postoperative ECMO (n) & $10(1.4)$ & $5(7.8)$ & .01 \\
\hline
\end{tabular}

TABLE 4. Risk factors for mortality

\begin{tabular}{lrrrrrr}
\hline & \multicolumn{2}{c}{$\begin{array}{c}\text { Univariable } \\
\text { analysis }\end{array}$} & & \multicolumn{2}{c}{$\begin{array}{c}\text { Multivariable } \\
\text { analysis }\end{array}$} \\
\cline { 2 - 3 } \cline { 6 - 7 } \multicolumn{1}{c}{ Factor } & HR & $\mathbf{9 5 \%}$ CI & & HR & $\mathbf{9 5 \% ~ C I ~}$ \\
\hline Use of preoperative inotropes & 3.59 & $2.09-6.17$ & & 2.59 & $1.43-4.68$ \\
Perioperative AT & 3.49 & $1.92-6.33$ & & 1.90 & $1.05-3.77$ \\
Use of postoperative inotropes & 2.37 & $1.07-5.22$ & & 1.90 & $0.79-4.57$ \\
Heterotaxy syndrome & 2.84 & $1.4-5.75$ & & 1.72 & $0.79-3.71$ \\
Single ventricle physiology & 2.62 & $1.59-4.33$ & & 1.64 & $0.91-2.96$ \\
Age $\leq$ 6 mo at surgery & 2.73 & $1.18-6.35$ & & 1.46 & $0.65-4.44$ \\
Hispanic & 1.73 & $1.05-2.85$ & & 1.46 & $0.86-2.46$ \\
RACHS-1 score $\geq 4$ & 3.15 & $1.84-5.41$ & & 1.45 & $0.8-2.65$ \\
\hline
\end{tabular}

$H R$, Hazard ratio; $C I$, confidence interval; $A T$, atrial tachycardia; $R A C H S-1$, Risk Adjustment for Congenital Heart Surgery.

(IQR, 7.0-24.7) in the non-AT group $(P=0.21)$. On multivariable analysis, perioperative AT was independently associated with a greater risk of mortality (Table 4). Within the study group, 8 patients died before discharge home; however, the mortality during the immediate 30 -day postoperative period was similar between the 2 groups. No statistically significant difference was seen in the risk of mortality between patients who developed AT preoperatively versus postoperatively $(P=0.25)$. The KaplanMeier survival curves for both groups and the log-rank test of significance are displayed in Figure 2.

\section{Antiarrhythmic Therapy}

A total of 57 patients $(89.1 \%)$ received inpatient antiarrhythmic therapy at the onset of AT. Propranolol was the most commonly used agent in $31(54.4 \%)$ patients, procainamide in $11(18.9 \%)$, esmolol in $7(12.1 \%)$, amiodarone in $5(8.8 \%)$, propranolol with digoxin in $2(3.5 \%)$, and metoprolol in $1(1.7 \%)$. Treatment failure occurred in 13 patients. The initial therapy with propranolol failed in 10 patients and required transition to sotalol in 6 patients and amiodarone in 4 patients. Esmolol therapy failed in 2

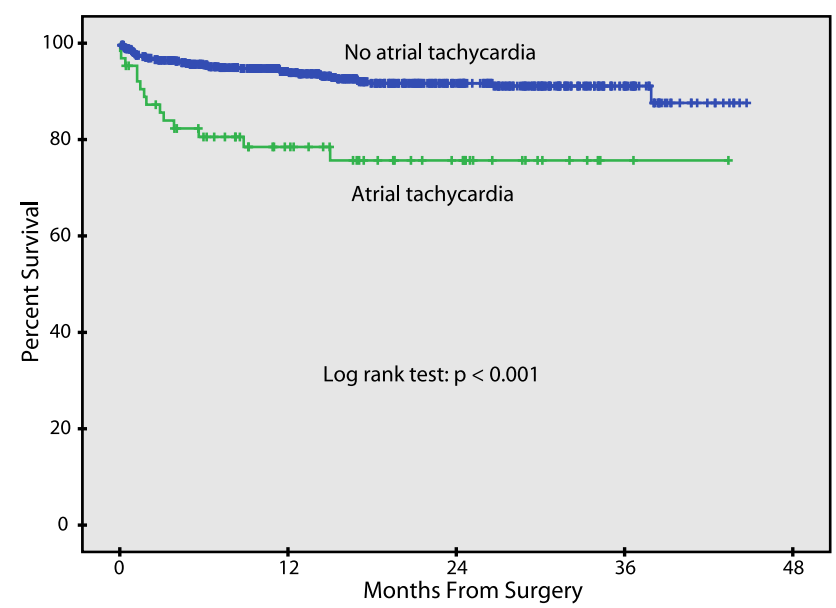

FIGURE 2. Kaplan-Meier survival curve of infants with perioperative atrial tachycardia compared with those without atrial tachycardia. 
patients, who were transitioned to procainamide, and procainamide therapy failed in 1 patient, who was transitioned to amiodarone. Treatment failure was not significantly associated with death $(P=0.26)$. The decision to not treat AT in 7 patients was predominantly because of the identification of reversible iatrogenic causes such as the presence of a deep central venous catheter or electrolyte derangement. One untreated patient died before discharge and the remaining 6 were successfully discharged with no antiarrhythmic therapy and no recurrence of AT to date.

Of the 56 survivors at hospital discharge, $48(85.7 \%)$ received outpatient antiarrhythmic therapy. Propranolol was again the most commonly used agent in 32 patients $(66.7 \%)$. Sotalol was used in $7(14.6 \%)$, amiodarone in 5 $(10.4 \%)$, propranolol with amiodarone in $2(4.2 \%)$, propranolol with digoxin in $1(2.1 \%)$, and metoprolol in 1 $(2.1 \%)$. Antiarrhythmic therapy was successfully discontinued in 23 patients $(41.0 \%)$ at a median follow-up of 14.1 months (IQR, 5.7-18.6) without recurrence.

\section{DISCUSSION}

In the present retrospective study of 777 infants undergoing cardiac surgery, perioperative AT developed in $8 \%$ of the infants and was independently associated with increased mortality, a finding not previously reported in the current literature. In the AT group, all-cause mortality was $21.9 \%$ compared with $7.2 \%$ in the control group during a median follow-up period of 16 months. Similar to a recent study by Trivedi and colleagues ${ }^{13}$ describing arrhythmias in children with hypoplastic left heart syndrome, we did not find a significant difference in 30-day mortality between the AT and control groups. This is in contrast to a previous investigation examining all arrhythmias. ${ }^{2}$ However, no study to date, including the 2 aforementioned reports, has examined AT and its associated mortality. An analysis of inpatient mortality did not reveal the cause of death to be directly attributable to AT. It would be difficult to ascertain whether AT directly led to death in patients with out-of-hospital events because their heart rhythm was not recorded at the event; however, it is unlikely that AT was the direct cause of death in these patients. We hypothesized that the association of AT with mortality is likely a surrogate marker of overall poor cardiovascular health and not a direct result of the arrhythmia.

A number of independent risk factors for developing AT were identified including age 6 months or younger at surgery, the use of 3 or more inotropes, transposition of the great arteries, total anomalous pulmonary venous return, and heterotaxy syndrome. Although Rosales and colleagues ${ }^{11}$ reported univariable associations between postoperative AT and both inotrope use and transposition of the great arteries, the present study is the first to report independent risk factors. Similar to previous studies, younger age continued to be a risk factor for ectopic automatic arrhythmias in infants from unknown mechanisms. ${ }^{1,2,6}$ The arrhythmogenic nature of inotropic agents and increased catecholamine levels might enhance atrial automaticity and thus providing the substrate to develop AT. Although the patient receiving 3 or more inotropes likely has morbidities beyond AT alone, those who develop AT with significant inotropic support likely represent an at-risk population of patients with comparably worse cardiovascular health. It is important to note that AT was associated with mortality, independent of all studied variables, including inotropic support. Thus, the patient receiving 3 or more inotropes who did not develop AT was at a decreased risk of death compared with those who did develop AT, suggesting that inotropes alone do not explain the increased risk of mortality in these patients. Although the use of epinephrine, calcium chloride, dopamine, and vasopressin in the perioperative period was associated with AT, the use of milrinone was not, in contrast to a recent report by Smith and colleagues. ${ }^{14}$ Thus, the combination of inotropes and the failing heart might manifest itself as AT, representing a marker of overall poor cardiovascular health and prognosis.

Specific diagnoses, including heterotaxy syndrome, were associated with AT. Heterotaxy syndrome is often associated with complex congenital heart disease and atrial isomerism. Previous studies have reported the prevalence of arrhythmias in both right ${ }^{15}$ and left ${ }^{16}$ atrial isomerism. Although the development of AT in these patients is likely multifactorial owing to the presence of associated anatomic cardiac lesions, the present study was not designed to explore the mechanistic etiologies of AT. Furthermore, the infants in the present study who developed AT did so at a median of 8.5 days postoperatively, which might be later than expected if the inciting factor responsible for the onset of AT was simply surgical trauma. This later time of onset might speak to an etiology and natural history different from trauma, such as inflammatory mechanisms, anatomic substrates, or a failing myocardium. Potential additional investigation might help to better elucidate the possible mechanistic etiologies for the development of AT, as well as possible therapeutic strategies to minimize untoward outcomes. The recognition and diagnosis of AT in this subgroup is difficult owing to the abnormal, multiform $\mathrm{P}$ waves. Therefore, vigilant observation of abnormal heart rates that acutely change, the "warm up and cool down" characteristics of the tachycardia, $\mathrm{P}$ wave morphology analysis, and atrioventricular conduction is needed to properly make the diagnosis.

AT was associated with significantly increased morbidity, including increased hospital length of stay and total inotrope duration. This is likely a factor of the infant's overall cardiovascular state and not directly attributable to AT alone. The 30-day readmission rate was not significantly different statistically between the 2 groups. Although the use of postoperative ECMO was more likely in the study 
group, AT was not the primary indication for ECMO in 4 of 5 patients.

In our cohort, AT was successfully treated with antiarrhythmic agents, as determined by follow-up Holter monitoring demonstrating no recurrent AT. Inpatient therapy with $\beta$-blockade, using propranolol and esmolol, was most commonly used, followed by procainamide and amiodarone. Treatment failure or the lack of treatment was not associated with death. Outpatient therapy was equally effective in the outpatient setting and can be successfully discontinued in most patients with a low risk of recurrence.

\section{Study Limitations}

Although this was the largest study of infants with perioperative AT to date, the study was limited by its singlecenter, retrospective design of a moderate number of patients. This sample size made it difficult to exclude the possibility of a type II error for other factors associated with mortality. Although overall mortality was worse in the AT group, the small sample size of the AT group was of limited statistical power to show a statistically significant difference in 30-day mortality between the 2 groups. In the subgroup analyses, considerations regarding the predictive risk factors were also of limited statistical power. The potential for selection bias also exists because sicker infants with closer monitoring might have had more frequent detection of AT than their healthier counterparts.

In conclusion, AT is an independent risk factor for mortality among infants undergoing cardiac surgery. It was observed during the perioperative period in $8 \%$ of infants undergoing cardiac surgery and was more common among infants aged 6 months or younger at surgery, those receiving 3 or more inotropes, and those with heterotaxy syndrome. Among the survivors, antiarrhythmic agents successfully controlled AT in most patients with a low recurrence risk after discontinuation. Additional study is needed to understand the influence of AT on outcome in this population.

\section{References}

1. Delaney JW, Moltedo JM, Dziura JD, Kopf GS, Snyder CS. Early postoperative arrhythmias after pediatric cardiac surgery. J Thorac Cardiovasc Surg. 2006;131: 1296-300.

2. Rekawek J, Kansy A, Miszczak-Knecht M, Manowska M, Bieganowska K, Brzezinska-Paszke M, et al. Risk factors for cardiac arrhythmias in children with congenital heart disease after surgical intervention in the early postoperative period. J Thorac Cardiovasc Surg. 2007;133:900-4.

3. Grosse-Wortmann L, Kreitz S, Grabitz RG, Vazquez-Jimenez JF, Messmer BJ, von Bernuth G, et al. Prevalence of and risk factors for perioperative arrhythmias in neonates and children after cardiopulmonary bypass: continuous Holter monitoring before and for three days after surgery. J Cardiothorac Surg. 2010; $5: 8$.

4. Pfammatter JP, Bachmann DC, Wagner BP, Pavlovic M, Berdat P, Carrel T, et al. Early postoperative arrhythmias after open-heart procedures in children with congenital heart disease. Pediatr Crit Care Med. 2001;2:217-22.

5. Pfammatter JP, Wagner B, Berdat P, Bachmann DC, Pavlovic M, Pfenninger J, et al. Procedural factors associated with early postoperative arrhythmias after repair of congenital heart defects. J Thorac Cardiovasc Surg. 2002;123:258-62.

6. Valsangiacomo E, Schmid ER, Schupbach RW, Schmidlin D, Molinari L, Waldvogel K, et al. Early postoperative arrhythmias after cardiac operation in children. Ann Thorac Surg. 2002;74:792-6.

7. Case CL, Gillette PC. Automatic atrial and junctional tachycardias in the pediatric patient: strategies for diagnosis and management. Pacing Clin Electrophysiol. 1993;16:1323-35.

8. Gillette PC. The mechanisms of supraventricular tachycardia in children. Circulation. 1976;54:133-9.

9. Gillette PC, Smith RT, Garson A Jr, Mullins CE, Gutgesell HP, Goh TH, et al. Chronic supraventricular tachycardia: a curable cause of congestive cardiomyopathy. JAMA. 1985;253:391-2.

10. Koike K, Hesslein PS, Finlay CD, Williams WG, Izukawa T, Freedom RM. Atrial automatic tachycardia in children. Am J Cardiol. 1988;61:1127-30.

11. Rosales AM, Walsh EP, Wessel DL, Triedman JK. Postoperative ectopic atrial tachycardia in children with congenital heart disease. Am J Cardiol. 2001;88: 1169-72.

12. Jenkins KJ, Gauvreau K, Newburger JW, Spray TL, Moller JH, Iezzoni LI. Consensus-based method for risk adjustment for surgery for congenital heart disease. J Thorac Cardiovasc Surg. 2002;123:110-8

13. Trivedi B, Smith PB, Barker PC, Jaggers J, Lodge AJ, Kanter RJ. Arrhythmias in patients with hypoplastic left heart syndrome. Am Heart J. 2011;161: 138-44.

14. Smith AH, Owen J, Borgman KY, Fish FA, Kannankeril PJ. Relation of milrinone after surgery for congenital heart disease to significant postoperative tachyarrhythmias. Am J Cardiol. 2011;108:1620-4.

15. Cheung YF, Cheng VY, Yung TC, Chau AK. Cardiac rhythm and symptomatic arrhythmia in right atrial isomerism. Am Heart J. 2002;144:159-64.

16. Wu MH, Wang JK, Lin JL, Lai LP, Lue HC, Hsieh FJ. Cardiac rhythm disturbances in patients with left atrial isomerism. Pacing Clin Electrophysiol. 2001; $24: 1631-8$. 